\title{
Adaptación y validez de la Escala de Personalidad Tipo D (DS-14) en una muestra mexicana
}

\author{
Adaptation and validity of the Type D Personality Scale (DS-14) in a Mexican sample
}

\author{
Arturo Juárez-García ${ }^{\mathrm{a}, *}$, César Merino-Soto ${ }^{\mathrm{b}}$, Javier Neri Uribe ${ }^{\mathrm{a}}$ \\ aUniversidad Autónoma del Estado de Morelos, México \\ bUniversidad de San Martín de Porres, Perú
}

\section{Resumen}

El objetivo del presente trabajo consistió en explorar las propiedades psicométricas de una adaptación al castellano del Cuestionario de Personalidad Tipo D (DS-14) en una muestra mexicana $(n=702)$. Se evaluó la escala mediante un enfoque no paramétrico basado en el escalamiento Mokken y se aplicó el análisis factorial confirmatorio por el método de estimación de mínimos cuadrados ponderados con media y varianza ajustada (WLSMV), consistencia interna con coeficiente omega $(\omega)$, invarianza de medición por género, y validez convergente con medidas de salud mental (MBI = burnout, GHQ = depresión, trastornos de sueño, disfunción social y síntomas somáticos), parcializando los efectos de estresores laborales (JCQ = demandas y control laboral). Los resultados evidenciaron correlaciones parciales de bajas a moderadas de los ítems DS-14 con los puntajes de salud mental, así como índices de confiabilidad aceptables ( $\omega$ $=.62-.86)$. Se halló invarianza de medición entre hombres y mujeres, y la estructura bifactorial original mostró índices de ajuste satisfactorios $(\mathrm{CFI}=.97$, RMSEA $=.04$, SRMR $=.05$ ). Adicionalmente, se vislumbró la viabilidad de una estructura unidimensional, en particular para una versión de 9 ítems (CFI = .99 , RMSEA $=.04$, SRMR $=.04$ ), la cual necesita estudios adicionales de replicabilidad. Se concluye que la escala DS-14 produce puntajes válidos y confiables, y se discuten estos hallazgos y sus implicaciones para la investigación futura.

Palabras clave: personalidad tipo D, DS-14, análisis confirmatorio, invarianza de medida, México.

\section{Para citar este artículo:}

Juárez-García, A., Merino-Soto, C., \& Neri, J. (2018). Adaptación y validez de la Escala de Personalidad Tipo D (DS-14) en una muestra mexicana. Liberabit, 24(2), 321-339. https://doi.org/10.24265/liberabit. 2018.v24n2.11

\begin{abstract}
The goal of this study was to explore the psychometric properties of a Spanish version of the Type D Personality Scale (DS-14) in a sample of Mexican participants $(n=702)$. The scale was evaluated through a non-parametric approach based on Mokken scaling. Moreover, confirmatory factor analyses were performed using the weighted least squares mean and variance (WLSMV) method, internal consistency estimation with omega coefficient $(\omega)$, measurement invariance by gender, and convergent validity with mental health measures (MBI = burnout, GHQ = depression, sleep disorders, social dysfunction, and somatic symptoms), and controlling job stressors (JCQ = job demands and job control). The results showed low to moderate partial correlations in DS-14 items concerning the mental health scores, and acceptable reliability rates $(\omega=.62-.86)$. Measurement invariance between men and women was found, and the original two-factor structure showed satisfactory adjustment indices (CFI $=.97$, RMSEA $=.04$, SRMR $=.05$ ). Furthermore, the viability of a one-dimensional structure, which needs future replicability studies, was glimpsed particularly in the 9-item version (CFI = .99, RMSEA $=.04$, SRMR $=.04)$. It is concluded that the DS-14 scale generates valid and reliable scores, and these findings and implications are discussed for further research.
\end{abstract}

Keywords: Type D personality, DS-14, confirmatory analysis, measurement invariance, Mexico.

Este es un artículo Open Access bajo la licencia Creative Commons Atribución-NoComercial-CompartirIgual 4.0

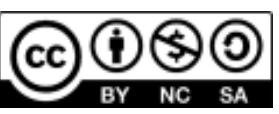




\section{Introducción}

El interés por identificar factores de personalidad asociados a problemas de salud no es algo nuevo, pues desde la Antigua Grecia, Galeno e Hipócrates clasificaban a la personalidad según el tipo de sangre (melancólico, colérico, flemático y sanguíneo) y la relacionaban a distintos males, lo que se vinculó claramente a posteriores teorías como la de Pavlov sobre la personalidad relacionada al tipo de sistema nervioso o la de Eysenck con los ejes extraversiónintroversión, neuroticismo-establidad emocional y psicotismo-autocontrol (Eysenck \& Eysenck,1987; Ruch, 1992; Stelmack \& Staikas, 1991). El mismo Eysenck (1967) identificó a los más introvertidos y emocionalmente inestables como los más vulnerables a problemas de salud, lo que se hizo extensivo a teorías de personalidad posteriores como el patrón de conducta tipo «A» o el modelo de los «Cinco Grandes» (Costa \& McCrae, 1990; Friedman \& Rosenman, 1959; Lahey, 2009).

En este contexto y de manera más reciente, Denollet et al. (1991, 1998) y Denollet et al. (1996), y con la influencia de teorías de Cloninger, Przybeck, Svrakic y Wetzel (1994) y Gray (1982), propuso la existencia de una tipología de personalidad o una disposición individual a experimentar y suprimir de manera simultánea el estrés emocional negativo que hace a las personas más vulnerables a problemas de salud, misma que llamó «personalidad tipo D» $(D=$ Disstressed $)$. La personalidad tipo $\mathrm{D}$ se entiende como aquella tendencia a experimentar las consecuencias del distrés crónico, caracterizada por la presencia simultánea e interrelación sinérgica de sus dos componentes: la afectividad negativa y la inhibición social (Denollet \& Conraads, 2011; Denollet, 2005).

Por un lado, la afectividad negativa se describe como una dimensión de carácter general de distrés subjetivo, integrada de una serie de estados de ánimo negativos relacionados con la ira, el nerviosismo, el miedo o la culpa, de modo tal que cuando se poseen bajos niveles de afectividad negativa se experimentaría en oposición, un estado representado por la serenidad, la tranquilidad y un ánimo positivo (Watson, Clark, \& Tellegen, 1988). Para Denollet (1998, 2000, 2005), los tres componentes claves de la afectividad negativa son la experimentación de sentimientos de irritabilidad, la disforia y la aprensión ansiosa (Tabla 1).

Por otro lado, la inhibición social implica inseguridad, tensión y retraimiento de circunstancias o interacciones sociales, así como la supresión de las emociones debido al miedo o rechazo de otros, lo que en general implica un comportamiento antisocial e inhibido ante situaciones que implican interacción social (Asendorpf, 1993). Denollet (1998, 2005) considera como componentes claves a incomodidad social, la reticencia y la falta de desenvolvimiento social (Tabla 1 ).

De esta forma, en la propuesta original de Denollet (2005), solo las personas que presentan altos puntajes en afectividad negativa e inhibición social de forma simultánea son clasificadas con personalidad tipo D (tipología bimodal-categórica), aunque ante la falta de claridad de sinergia entre ambos constructos, algunos estudios han optado por la suma global de los puntajes de ambos constructos (dimensionalidad continua) (De Fruyt \& Denollet, 2002; Horwood, Anglim, \& Tooley, 2015; Montero, Bermúdez, \& Rueda, 2017).

La relevancia de la personalidad tipo $\mathrm{D}$ en el ámbito biopsicosocial de la salud se sustenta en un creciente cúmulo de evidencias, que incluyen principalmente su relación con la morbilidad y mortalidad cardiovascular (Denollet, 1991,1998; Denollet et al., 1996; Denollet \& Conraads, 2011; Denollet, Vaes, \& Brutsaert, 2000; Denollet, Pedersen, Vrints, \& Conraads, 2006), con la ansiedad, la depresión, el inadecuado ajuste psicológico y la salud mental en general (Mols, Thong, van de Poll, Roukema, \& Denollet, 2012; Montero, Rueda, \& Bermúdez, 2012; Pedersen, Domburg, Thenus, Jordaens, \& Erdman, 2004), con el insomnio (Akram et al., 2018) y con un impacto negativo en general en la calidad de vida de pacientes en seguimiento (Mols 
et al., 2012; Pedersen \& Denollet, 2003; Pedersen, Theuns, Muskens-Heemskerk, Erdman, \& Jordaens, 2007). Se han propuesto diversos mecanismos fisiológicos mediadores que explican su impacto en la salud, que incluyen el rol de biomarcadores inmunológicos y citosinas proinflamatorias (Conraads et al., 2006; Denollet et al., 2003), de biomarcadores neuroendócrinos (Habra, Linden, Anderson, \& Weinberg, 2003; Sher, 2005; Whitehead, PerkinsPorras, Strike, Magid, \& Steptoe, 2007), de reactividad cardiovascular (Williams, O’Carrol, \& O’Connor, 2009), o de la adopción de ciertos estilos de vida (Williams et al., 2008).

Aunque muchos estudios han sido realizados originalmente en Bélgica por Denollet (2005), hallazgos de la misma naturaleza se han extendido a muestras norteamericanas (Barnett, Ledoux, Garcini, \& Bakker, 2009), chinas (Lin et al., 2017), alemanas (Jandackova, Koening, Jarczok, Fischer, \& Thayer, 2017), danesas (Spindler, Kruse, Zwisler, \& Pedersen, 2009), irlandesas e inglesas (Williams et al., 2008), islandesas (Svansdottir et al., 2012), españolas (Montero et al., 2017; Montero et al., 2012), entre otras; habiendo estudios transculturales en más de 22 países (Kupper et al., 2013), lo que significa un avance importante en el ámbito internacional sobre la existencia de una personalidad tipo D con riesgo a la salud.

Para la medición de la personalidad tipo D primero se desarrolló un cuestionario de 24 ítems con preguntas inspiradas en el MMPI (Denollet, 1991; Denollet et al., 1996; De Fruyt \& Denollet, 2002), que luego se transformó en 16 ítems (Denollet, 1998, 2000), para llegar a una versión final de 14 ítems (DS14) repartidos en las dos dimensiones de afectividad negativa e inhibición social (Denollet, 2005).

En cuanto a sus propiedades psicométricas, desde el primer análisis factorial exploratorio documentado en la versión de 16 ítems en 400 participantes belgas, se encontraron 2 factores correspondientes a afectividad negativa e inhibición social, con confiabilidades alfa de Cronbach de .89 y .92, respectivamente (Denollet, 1998). Posteriormente, se confirmó esto en una muestra de 225 pacientes daneses con confiabilidades de .83 y .76; sin embargo, desde aquí, se empezó a visualizar la necesidad de una versión de 14 ítems (Pedersen \& Denollet, 2004). De esta forma la escala de personalidad tipo D de 14 ítems (DS-14), se consolida con un estudio de 3678 participantes, donde se mantuvieron los altos niveles de confiabilidad (.88 y .86) y la estructura bidimensional en tres muestras: pacientes coronarios, hipertensos, y en población en general (Denollet, 2005). Estudios factoriales exploratorios con resultados similares se han confirmado en Alemania (Grande et al., 2004), Lithuania (Bunevicius et al., 2013) y en Taiwan (Weng et al., 2013).

Aunque se han realizado pocos estudios con análisis factoriales confirmatorios, estos han mostrado también la estructura bifactorial esperada, con coeficientes alfa de Cronbach satisfactorios en ambas dimensiones $(>.80)$, en pacientes con distintos malestares cardiacos (Spindler et al., 2009; Svansdottir et al., 2012) y con dolor crónico (Barnett et al., 2009).

En el idioma español también se han analizado las propiedades psicométricas del cuestionario DS-14, aunque solo en el estudio efectuado por Montero et al. (2017), a partir de una muestra compuesta por 890 participantes, donde se incluyeron personas sanas ( $n$ $=430)$, pacientes con cardiopatía isquémica $(n=230)$ y oncológicos $(n=230)$. Los resultados de los análisis factoriales exploratorios y confirmatorios realizados demostraron una estructura compuesta por dos dimensiones acorde a la original; se obtuvieron, además, asociaciones positivas con medidas de agotamiento vital, malestar emocional, ansiedad, depresión e ira interna, y negativas con apoyo social, satisfacción con la vida y extroversión, así como coeficientes alfa de Cronbach satisfactorios (entre .81 y .90 para las tres muestras).

El cúmulo de investigación descrito antes denota la relevancia de la personalidad tipo D en la salud 
biopsicosocial en general, sin embargo, existen algunos estudios que ponen en juicio su validez como por ejemplo su incapacidad para detectar riesgo de mortalidad en pacientes cardiacos (Coyne et al., 2011) y su independencia de los factores de personalidad de neuroticismo e introversión (Horwood et al., 2015). En el ámbito psicométrico y metodológico en particular, se puede agregar, la falta de claridad en la forma de interacción sinérgica entre afectividad negativa e inhibición social, que parece ser más unidimensional que bidimensional (Ferguson et al., 2009), la falta de estudios en poblaciones sanas (Mols \& Denollet, 2010), el control metodológico de variables psicosociales del contexto que se relacionan de antemano con problemas de salud mental (e.g. psicosociales laborales) (Madsen et al., 2017; Stansfeld \& Candy, 2006; Theorell et al., 2015) y el hecho de que el estudio de las propiedades psicométricas de la escala DS-14 se ha dado principalmente en poblaciones europeas y en países desarrollados, incluyendo las traducciones al castellano de España, que pueden tener diferencias relevantes al significado semántico y de lenguaje latinoamericano en la evaluación psicométrica, por lo que son necesarios estudios y adaptaciones en otras latitudes, específicamente en Latinoamérica y en México en particular.

Adicionalmente, vale la pena señalar que el análisis de invarianza factorial es prácticamente ausente en el estudio de la escala de personalidad tipo D, encontrándose solo el estudio de Kupper et al. (2013) que confirma la invarianza configuracional, métrica y escalar entre distintas regiones europeas, y el de Emons, Meijer, y Denollet (2007) que prueba la ausencia del funcionamiento diferencial entre personas clínicamente enfermas y sanas, lo que significa un campo fértil de exploración en variables sociodemográficas, en particular el género, que por sus implicaciones en la formación de la personalidad tipo D y su impacto diferencial en los mecanismos de enfermedad cardiovascular y los trastornos mentales, pueden estar relacionados a la eficacia de su medida. Por ejemplo, se sabe que sí existen efectos diferenciales de la personalidad tipo D según el sexo (Gramer, Haar, \& Mitteregger, 2018), y que existen diferencias en los mecanismos del proceso saludenfermedad mental entre hombres y mujeres (Rosenfield \& Mouzon, 2013) y, no menos importante, se ha sugerido una investigación más justa en el tratamiento independiente de géneros en estudios interdisciplinares (Messing et al., 2003), por lo que resulta indispensable la valoración de la invarianza de medición del DS-14 entre hombres y mujeres.

Por otro lado, se sabe que la afectividad negativa en combinación con la inhibición social implican algunos mecanismos de neuroticismo-introversión que se vinculan a distintos problemas de salud mental, tales como estados de ánimo depresivos, problemas de sueño, somatizaciones y problemas de funcionamiento social en general (Mols et al., 2012; Montero et al., 2012 ), así como su relación con el agotamiento emocional o burnout (Armon, 2014; Polman, Borkoles, \& Nicholls, 2010), lo que confirma su idoneidad como criterios de validación en estudios psicométricos de la escala DS-14.

Dado lo anterior, el objetivo del presente trabajo consistió en explorar las propiedades psicométricas de una adaptación del cuestionario DS-14 en una muestra de trabajadores mexicanos, incluyendo su estructura factorial, su consistencia interna, su invarianza de medición entre hombres y mujeres y su validez convergente con medidas de salud mental.

\section{Método}

Se trató de un estudio de tipo instrumental (Montero \& León, 2007) en el que se buscó comprobar la estructura de la escala (afectividad negativa e inhibición social) mediante el análisis factorial confirmatorio (AFC), probar su invarianza factorial entre grupos según sexo y estimar su consistencia interna. Asimismo, se verificó su validez convergente estimando su correlación con los constructos de salud mental (depresión, trastornos de sueño, disfunción social y síntomas somáticos) y 
síndrome de burnout (agotamiento emocional, indiferencia y eficacia profesional), parcializando los efectos de contexto laboral por control metodológico (Aneshensel, 2012), en particular, las demandas psicológicas y el control laboral, los cuales han mostrado un impacto relevante en la salud mental independientemente de la personalidad (Madsen et al., 2017; Stansfeld \& Candy, 2006; Theorell et al., 2015).

\section{Participantes}

Algunas revisiones (Costello \& Osborne, 2005) señalan entre las reglas de tamaño de muestra más exigentes una relación de hasta 20:1 entre personas e ítems, lo que significa que para la escala DS-14 son necesarios al menos 280 participantes. Como parte de un proyecto de salud ocupacional más grande y mediante un muestreo no aleatorio y por conveniencia participaron de manera voluntaria 702 personas, de las cuales 533 eran trabajadores informales ubicados en tres organizaciones de comercio ambulante y establecimientos fijos en zonas urbanas, y 160 eran trabajadores formales de dos organizaciones, todos ubicados en la ciudad de Cuernavaca, Morelos, México. El 60.4\% eran hombres y 38.0\% mujeres, con una media de edad de 34.77 años $(D E=11.34)$. 40.7\% eran casados, 32.3\% solteros, $20.8 \%$ de unión libre y $5.6 \%$ divorciados o viudos. La mayoría tenía escolaridad media básica superior (secundaria) (34.6\%), seguido por bachillerato terminado (28.3\%) y licenciatura (16.7\%).

\section{Responsabilidad ética}

Esta investigación siguió los requerimientos éticos de la Declaración de Helsinki (2013) en relación a la investigación con seres humanos, respetando la autonomía, asegurando la maximización de beneficiencia sobre cualquier riesgo, buscando la justicia distributiva de participación y la confidencialidad y privacidad de la información. Se utilizó un formato de consentimiento informado con base en el sugerido por el comité de ética nacional IRB (international review board) de Estados Unidos de Norteamérica.

\section{Instrumentos}

Escala de Personalidad Tipo D. Se realizó la adaptación y traducción de la escala desarrollada por Denollet (2005) versión de 14 ítems (DS-14) por dos investigadores bilingües, de acuerdo a sugerencias metodológicas internacionales (Beaton, Bombardier, Guillemin, \& Ferraz, 2000). En un primer paso, se realizó el proceso de traducción-retraducción, donde uno de los especialistas hizo la traducción del inglés al castellano (lengua materna); en un segundo paso, con esa versión se hizo lo opuesto. En seguida, ambos investigadores discutieron, analizaron e hicieron ajustes hasta llegar a una versión que luego fue discutida y retroalimentada por el equipo de investigación (4 personas con experiencia en factores psicosociales), para llegar así a una versión final. La escala incluyó los componentes originales de afectividad negativa e inhibición social con cuatro opciones de respuesta que van desde 0 (totalmente falso), 1 (más o menos falso), 2 (neutral), 3 (más o menos cierto), hasta 4 (totalmente cierto) (ver Tabla 1).

Cuestionario General de Salud (GHQ por sus siglas en inglés). Se utilizó la versión de 28 ítems validada en México por Medina-Mora et al. (1983), en la que se obtuvieron cuatro dimensiones en análisis factoriales que explicaron el $41 \%$ de la varianza (síntomas somáticos, trastornos de sueño, disfunción social y depresión), las cuales además correlacionaron con valoraciones clínicas de salud mental independientes, mostrando coeficentes entre $r=.25$ y $r=.62$, y una sensibilidad y especificidad de $72 \mathrm{y}$ $73 \%$, respectivamente. Su diseño incluye cuatro opciones de respuesta que variaron desde «no» o «más que antes» (1) hasta «sí», «mucho menos que antes» o «mucho más que antes» (4) según fuera el ítem, y a mayor puntaje implica mayor deterioro en la salud mental.

Maslach Burnout Inventory General Survey (MBI-GS). Con autorización de la editorial Mind Garden ${ }^{\circledR}$ (Maslach, Jackson, \& Leiter, 2010), se utilizó la versión de 16 ítems validada para población latinoamericana (Juárez et al., 2018), que mide los 
constructos de agotamiento emocional, indiferencia y eficacia profesional, en seis opciones de respuesta que van desde nunca (0) hasta todos los días (6). Los valores de consistencia interna $(\omega=.03-.86)$ y la estructura trifactorial original mostró indices de ajuste satisfactorios (CFI > .99; RMSEA < .04).

\section{Cuestionario del Contenido del Trabajo (JCQ por} sus siglas en inglés). Para efectos de parcialización de otras variables relevantes en la esperada correlación entre la personalidad tipo D y la salud mental, se evaluaron las dimensiones de demandas psicológicas y control laboral. Se utilizó la versión de 16 ítems que incluye las dimensiones de demandas (6 ítems) y control laboral (9 ítems) con 4 opciones de respuesta que van desde totalmente en desacuerdo (1) hasta totalmente de acuerdo (4). Fue validado en México previamente (González, Juárez, Camacho, Noriega, \& Escobedo, 2015) con indices de ajuste adecuados (CFI = .91; RMSEA $=.05)$ y consistencia interna aceptable $(\alpha=$ .71 para demandas y $\alpha=.72$ para control).

\section{Procedimiento}

Se gestionaron permisos con autoridades de tres organizaciones informales y dos formales y se realizaron invitaciones individuales a cada trabajador, quienes respondieron los cuestionarios en los espacios libres a lo largo de la jornada laboral en su lugar de trabajo. Respecto a la estrategia de análisis de resultados, en primer lugar, se evaluaron las presunsiones básicas del puntaje del instrumento mediante un enfoque no paramétrico de teoría de respuesta al ítem, específicamente el escalamiento Mokken (1997) aplicado para datos politómicos (Molenaar, 1997). Este modelo asume que el puntaje de interés se usará en el nivel ordinal, y que la forma funcional de la relación entre la respuesta a un ítem y el puntaje del atributo es monotónica, sea lineal o no lineal. Este procedimiento puede ser considerado un precursor para el modelamiento paramétrico de otros modelos de teoría de respuesta al ítem (Van der

\section{Tabla 1}

Contenido del DS-14

\begin{tabular}{|c|c|c|}
\hline Item & Contenido & Constructo base \\
\hline \multicolumn{3}{|c|}{ Afectividad Negativa } \\
\hline 2 & Frecuentemente hago «dramas» por cosas sin importancia & Aprensión ansiosa \\
\hline 4 & Frecuentemente me siento infeliz & Disforia \\
\hline 5 & Frecuentemente me siento irritado & Irritabilidad \\
\hline 7 & Veo las cosas con pesimismo & Disforia \\
\hline 9 & Frecuentemente estoy de mal humor & Irritabilidad \\
\hline 12 & Frecuentemente estoy preocupado por algo & Aprensión ansiosa \\
\hline 13 & Frecuentemente estoy con «las pilas bajas» & Disforia \\
\hline \multicolumn{3}{|c|}{ Inhibición social } \\
\hline 1 & $\begin{array}{l}\text { Puedo entrar en contacto fácilmente cuando } \\
\text { conozco a personas nuevas }\end{array}$ & Desenvolvimiento social (recodificar) \\
\hline 3 & Frecuentemente hablo con extraños & Desenvolvimiento social (recodificar) \\
\hline 6 & $\begin{array}{l}\text { Frecuentemente me siento inhibido en situaciones } \\
\text { de convivencia con otras personas }\end{array}$ & Incomodidad en situaciones sociales \\
\hline 8 & Me es muy difícil iniciar una conversación & Incomodidad en situaciones sociales \\
\hline 10 & Soy una persona muy introvertida & Reticencia \\
\hline 11 & Prefiero tratar a las personas con cierta distancia & Reticencia \\
\hline 14 & Cuando socializo, no encuentro las cosas correctas de que hablar & Incomodidad en situaciones sociales \\
\hline
\end{tabular}


Ark, 2007). El cumplimiento de estos criterios forma el modelo monotónicamente homogéneo (Mokken, 1997).

Una escala que satisface el modelo de Mokken (1997) debe cumplir con los criterios de unidimensionalidad, monoticidad e independencia local, los cuales son condiciones suficientes para interpretacion ordinal de los puntajes de un instrumento (Stochl, Jones, \& Croudace, 2012). El criterio principal es la unidimensionalidad, e indica que una sola dimensión latente explica la relación entre los ítems de una medida. La monoticidad se refiere a la forma incremental en que la respuesta a un ítem se relaciona con el atributo, ambos variando de manera creciente. La independencia local se refiere a la suficiencia del atributo latente (constructo) para influenciar en la respuesta a un ítem, y no requiriendo la información del resto de ítems. El cumplimiento de estos criterios forma el modelo monotónicamente homogéneo (Mokken, 1997), pero puede ser visto como la condición necesaria para alguna medida psicométrica construida sobre un conjunto de ítems. Un concepto central en este procedimiento es la escalabilidad, evaluada mediante el coeficiente $H$ de Loevinger, en que valores menores a .30 indican pobre o ninguna escalabilidad del ítem, y una buena magnitud de escalabilidad es $>.50$ (Hemker, Sijtsma, \& Molenaar, 1995). Este coeficiente está vinculado con las condiciones anteriores es un indicador global de la calidad de la medicion (Mokken, 1997); en este sentido, para propósitos del presente estudio, la monoticidad será aproximada por este coeficiente, y la unidimensionalidad y la independencia local fueron corroborados por el enfoque de modelos de ecuaciones estructurales (SEM). El último criterio es equivalente a la correlación de los residuales en el modelamiento con enfoque SEM (Kline, 2010; Stochl et al., 2012).

En un segundo paso, se aplicó el análisis factorial confirmatorio por medio SEM para verificar la dimensionalidad y la validez de los ítems en relación a su constructo, los errores correlacionados entre los ítems, la relación entre los constructos y la confiabilidad de consistencia interna. Este procedimiento se aplicó para avanzar en el examen de la estructura interna del DS-14 desde un enfoque lineal, que usualmente forma parte de la validación psicométrica de la escala de personalidad tipo D (e.g., Denollet, 1998, 2005; Montero et al., 2017). Para esto, se aplicó un estimador robusto para variables categóricas (Muthén, 1984), basado en ajustar el primer y segundo momento del estadístico $\chi^{2}$ (meanand-variance-adjusted unweighted least squares) (WLSMV, Asparouhov \& Muthén, 2010). Este método usa un vínculo probit para modelar la relación funcional entre los ítems y el constructo, además de usar correlaciones policóricas inter-ítems, y estimación de umbrales (thresholds) entre categorías de respuestas. Este estimador tiende a obtener parámetros (e.g., cargas factoriales) más precisos, particularmente cuando la asimetría distribucional de los ítems es fuerte (Li, 2016; Sass, Schmitt, \& Marsh, 2014).

El presente estudio consideró usar niveles más estrictos de evaluación del modelamiento: cargas factoriales iguales o mayores a .50 e índices de ajuste con CFI $\geq .97$, RMSEA $\leq .04$, y SRMR $\leq .04$. Para la estimación de consistencia interna se calculó el coeficiente omega $(\omega)$, un coeficiente de confiabilidad más preciso que alfa de Cronbach $(\alpha)$ cuando no se cumplen los presupuestos de este (Dunn, Baguley, \& Brunsden, 2014).

Para examinar la invarianza de medición, se aplicó el análisis factorial de grupo múltiple (multiple group confirmatory factor analysis, MGFCA; Byrne, 2008; Byrne, Shavelson, \& Muthén, 1989), con una estrategia secuencial de modelos que variaron en la magnitud de las restricciones; mediante esto, primero se probó un modelo sin restricciones (invarianza configuracional), y luego otros más restringidos, como la igualdad de las cargas factoriales (invarianza métrica o débil) de los umbrales o thresholds (invarianza escalar o fuerte) y de los residuales (invarianza estricta). Las diferencias en el CFI de los modelos 
comparados menores a .01 (Putnick \& Bornstein, 2016) fueron considerados como criterio de invarianza.

Posteriormente, se utilizaron correlaciones de orden cero para verificar la validez de los ítems del DS-14 con criterios externos de salud mental (dimensiones de burnout y salud mental), y para controlar los efectos de estresores laborales y otras variables sociodemográficas relevantes, se estimó el coeficente de segundo orden o de corelación parcial, una opción metodológica para descartar efectos espúreos (Aneshensel, 2012). La identificacion de correlaciones de orden cero y parciales y su clasificación de bajas, moderadas o altas, estuvo basada en el criterio empírico propuesto por Bosco, Aguinis, Singh, Field y Pierce (2015).

\section{Resultados}

Análisis preliminar. Ya que se decidió reemplazar ítems no respondidos, para reducir la cantidad de respuestas imputadas y su probable impacto sobre el análisis estadístico, se eliminaron participantes con más de 3 ítems sin responder. Con esta decisión, se removieron 12 participantes con $14(n=3, .4 \%)$ hasta 3 ítems no respondidos $(n=3, .4 \%)$. También para reducir la probabilidad de respuestas irrelevantes, específicamente la misma respuesta a todos los ítems, se eliminaron 3 participantes cuyas respuestas usaron las mismas 0, 1 y 2. En conjunto, se eliminaron 15 participantes, reduciéndose la muestra a 687 personas. El método de imputación fue la respuesta modal en cada ítem, aplicándose a 60 sujetos (8.5\%) con uno o dos ítems no respondidos.

\section{Análisis de la dimensionalidad}

Enfoque no paramétrico. En las subescalas (Tabla 2), se observó un mejor escalamiento de Afectividad Negativa (AN) comparado con Inhibición Social (IS), aunque fue necesario remover ítems en ambas subescalas. Específicamente, en AN el ítem 4 no alcanzó el mínimo nivel $\left(\mathrm{H}_{\mathrm{j}}>\right.$.30), así que luego de ser removido, la escalabilidad del puntaje AN se elevó sustancialmente. Debido que en esta fase se buscó maximizar la escalabilidad de AN, se eligió remover el siguiente ítem con $\mathrm{H}_{\mathrm{j}}$ comparativamente bajo, de tal modo que el ítem 4 fue el siguiente candidato para remover. El coefciente $\mathrm{H}_{\mathrm{j}}$ final para el puntaje AN fue mayor a .50, y fue considerado satisfactorio.

En la subescala IS, el primer paso detectó claramente que los ítems 1 y 3 no convergían con un apropiado escalamiento dentro del puntaje IS. La remoción secuencial en el paso 2 y 3 mejoraron el escalamiento de IS, pero aún fue menor a .40; un paso adicional con la remoción del ítem 10 fue requerido. El resultado final para esta subescala fue que todos sus items mostraron $\mathrm{H}_{\mathrm{j}}>.40$.

Modelamiento lineal (análisis factorial confirmatorio). Los modelos evaluados aparecen en la Tabla 3. Dadas las altas correlaciones entre las dimensiones de inhibición social y afectividad negativa $(>.50)$ encontradas en este estudio y otras investigaciones previas, se decidió probar también el modelo unidimensional en conformidad incluso con estudios del mismo autor de la escala (De Fruyt \& Denollet, 2002), donde se usa el puntaje total como un indicador continuo para su interpretación. Sus índices de ajuste fueron satisfactorios, y fueron parecidos al modelo de dos factores correlacionados (modelo original), incluso las cargas factoriales de ambos tambien fueron similares. Por otro lado, el modelo de errores correlacionados entre los ítems 1 y 3, trató de modelar la varianza no asociada a los factores AN e IS; el ajuste de este modelo fue mejor que los dos modelos anteriores. La correlación residual entre los ítems 1 y 3 fue .345 ( $\mathrm{z}=8.85, p<.01)$. En el modelo sin los ítems recodificados (1 y 3 ), el ajuste fue notoriamente mejor, sugiriendo que la remoción de estos ítems causa un aumento significativo del ajuste. Finalmente, se probó el modelo derivado de la aplicación del análisis del escalamiento de Mokken; que se conformó de manera unidimensional por 9 ítems (5, 7, 9, 12, 13, 6, 8,11 y 14) (Tabla 3). El ajuste de este modelo fue tan bueno como el modelo 
Tabla 2

Escalamiento Mokken: coeficiente H para ítems $\left(H_{j}\right)$ y puntajes escalares $(H)(n=687)$

\begin{tabular}{|c|c|c|c|c|c|c|}
\hline & Paso 1 & Paso 2 & Paso 3 & Paso 4 & Puntaje total & Puntaje final \\
\hline \multicolumn{7}{|c|}{ Afectividad Negativa (AN) } \\
\hline 2DPER & $.394 * *$ & $.439 * *$ & - & - & .286 & - \\
\hline 4DPER & $.266 * *$ & - & - & - & .176 & - \\
\hline 5DPER & $.479 * *$ & $.509 * *$ & $.506 * *$ & - & $.335 * *$ & $.430 * *$ \\
\hline 7DPER & $.427 * *$ & $.460 * *$ & $.470 * *$ & - & $.321 * *$ & $.424 * *$ \\
\hline 9DPER & $.467 * *$ & $.516 * *$ & $.540 * *$ & - & $.364 * *$ & $.476 * *$ \\
\hline 12DPER & $.430 * *$ & $.478 * *$ & $.492 * *$ & - & $.322 * *$ & $.431 * *$ \\
\hline 13DPER & $.471^{* *}$ & $.525 * *$ & $.551 * *$ & - & $.356 * *$ & $.481 * *$ \\
\hline Total AN & .415 & $.488 * *$ & $.512 * *$ & - & - & \\
\hline Inhibición Social (IS) & Paso 1 & Paso 2 & Paso 3 & Paso 4 & & \\
\hline 1DPER & .149 & .103 & - & - & .067 & - \\
\hline 3DPER & .013 & - & - & - & -.080 & - \\
\hline 6DPER & $.257 * *$ & $.342 * *$ & $.403 * *$ & $.434^{* *}$ & $.328 * *$ & $.423 * *$ \\
\hline 8DPER & $.276 * *$ & $.347 * *$ & $.403 * *$ & $.438 * *$ & $.281 * *$ & $.367 * *$ \\
\hline 10DPE & $.206 * *$ & $.268 * *$ & $.326 * *$ & - & $.259 * *$ & - \\
\hline 11DPE & $.304 * *$ & $.365 * *$ & $.410 * *$ & $.417 * *$ & $.323 * *$ & $.399 * *$ \\
\hline 14DPE & $.287 * *$ & $.368 * *$ & $.430 * *$ & $.465 * *$ & $.332 * *$ & $.439 * *$ \\
\hline Total IS & $.211^{* *}$ & $.301 * *$ & $.395 * *$ & $.439 * *$ & & \\
\hline Escala total & - & - & - & - & $.258 * *$ & $.430 * *$ \\
\hline
\end{tabular}

previo (sin los ítems recodificados), e incluso estadísticamente mejor. El modelo final elegido fue el correspondiente con los ítems elegidos en el modelamiento Mokken.

Las cargas factoriales de los ítems se mantuvieron elevadas, excepcionalmente bajas en los ítems 1 y 3 de IS, y moderadamente bajas en los ítems 2 y 4 del factor AN. El ítem 10 de IS tendió a mostrar cargas factoriales moderadas en todos los modelos evaluados. Por otro lado, destacó que la correlacion entre los factores AN e IS se mantuvo superior a .80 en todos los modelos de dos factores evaluados.

La confiabilidad obtenida por el coeficiente omega (línea inferior de la Tabla 3), fue satisfactoria para los puntajes sin los ítems 1 y 3 del componente IS; el puntaje para la versión undimensional final (9 ítems) mostró adecuado nivel de confiabilidad, siendo inlcuso ligeramente el más alto que el resto.
Invarianza de medición. Se evaluó la invarianza de medición comparando varones y mujeres, y para ello se usó el modelo unidimensional final de nueve ítems. Se estableció el modelo configuracional en ambos grupos, y luego se ajustó el modelo de invarianza débil o igualdad de cargas factoriales. La diferencia entre ambos modelos fue $10.3647\left(\Delta \mathrm{gl}=8, \Delta_{\text {CFI }}=.001\right)$, $\mathrm{y}$ no fue estadísticamente significativa. En el siguiente paso de la invarianza, invarianza fuerte, la diferencia $\Delta \chi^{2}$ entre este modelo y el anterior fue $3.2415(\Delta \mathrm{gl}=8$, $\left.\Delta_{\text {CFI }}=.002\right)$ y tampoco fue estadísticamente significativa. Finalmente, se probó la invarianza estricta y la diferencia entre este modelo y el anterior no fue estadísticamente significativa $\left(\Delta \chi^{2}=7.8583, \Delta \mathrm{gl}=9\right.$, $\left.\Delta_{\text {CFI }}=.001\right)$. De acuerdo a estos resultados, el constructo unidimensional derivado mantiene altos niveles de invarianza de medición entre sexos. 


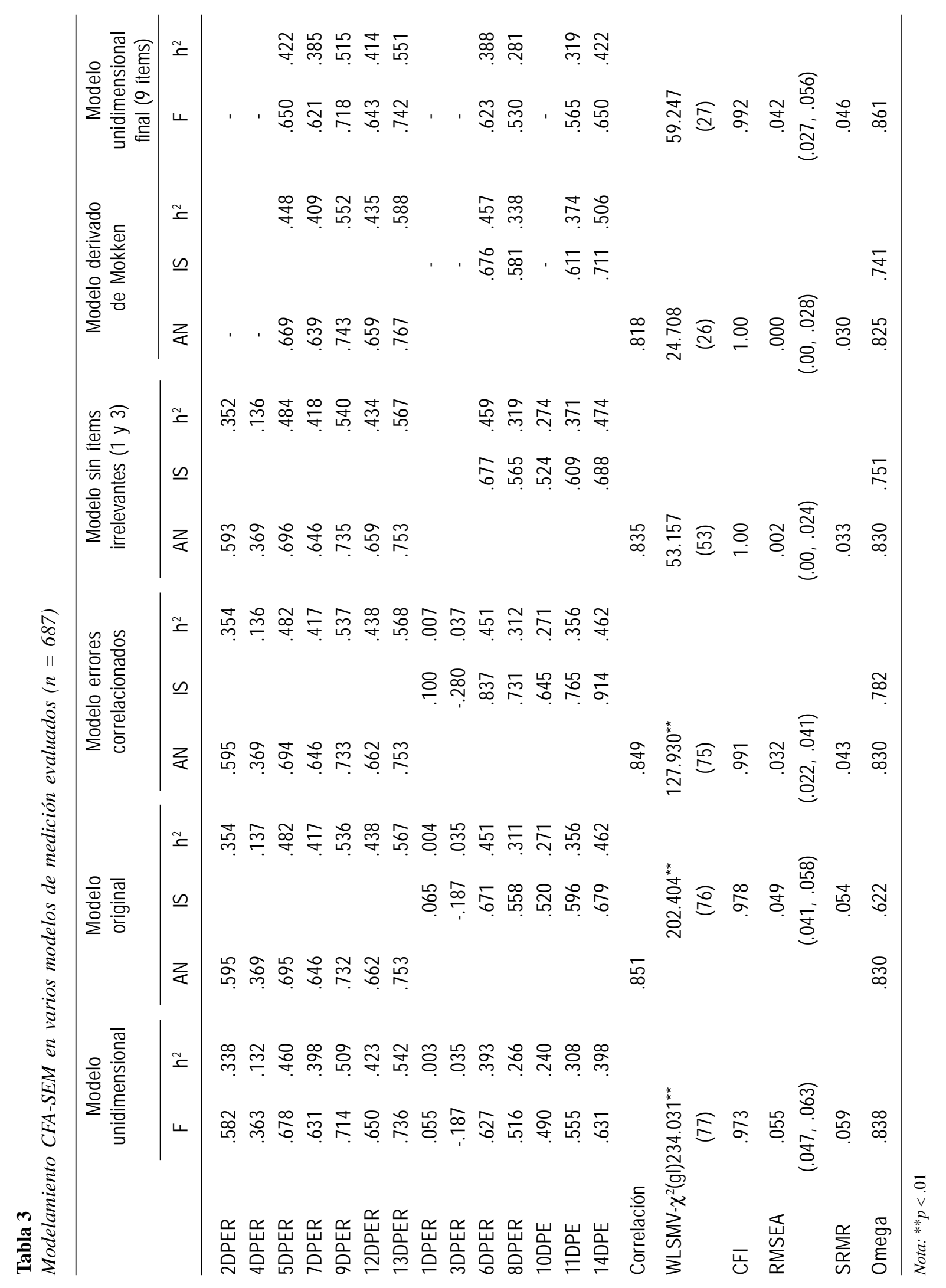




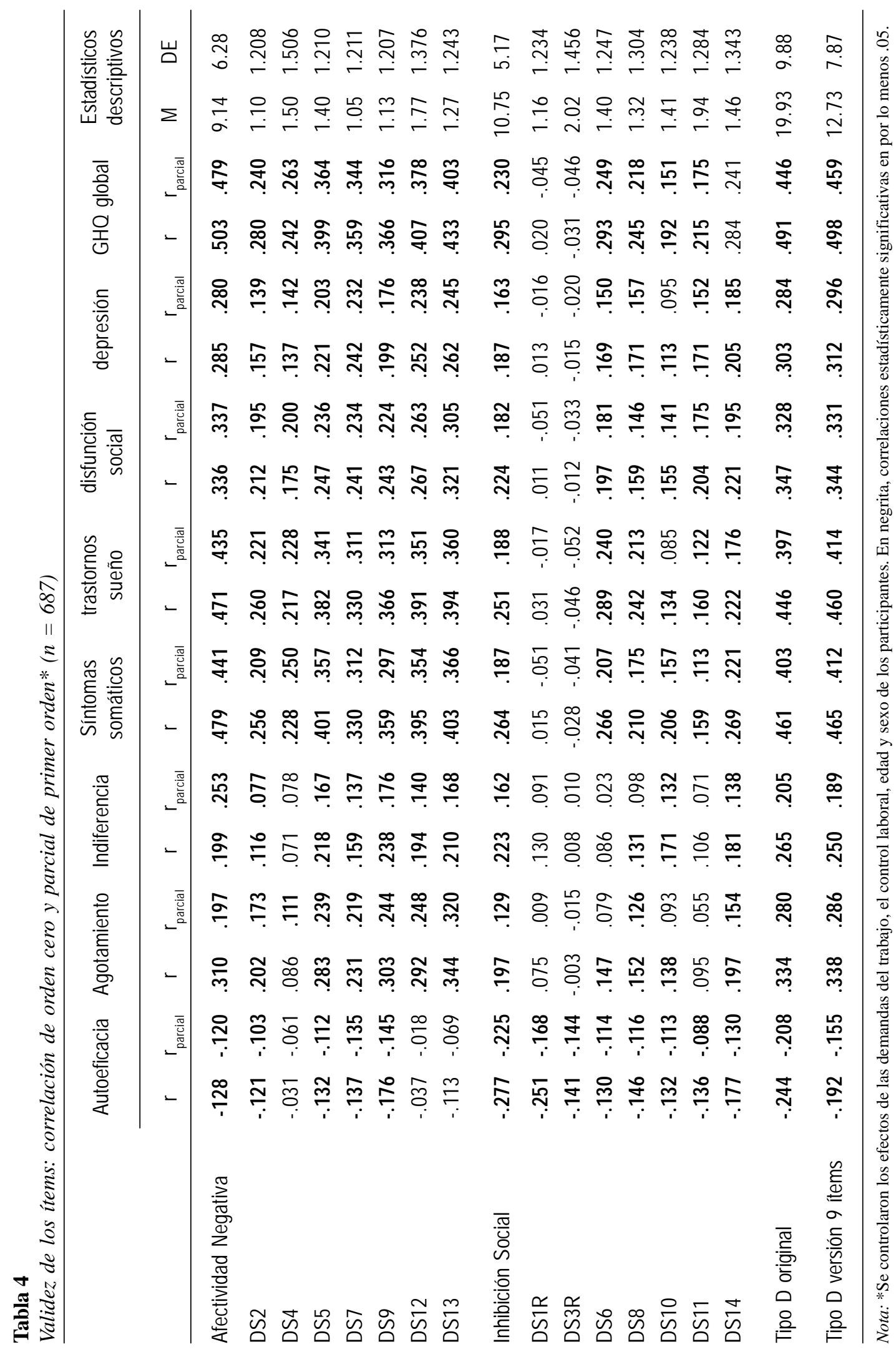


Validez externa de los ítems. Las correlaciones de los ítems del DS-14 con los puntajes del MBI-GS y GHQ-28 generalmente fueron de baja o moderada magnitud (Tabla 4). Con Eficacia Profesional se observaron, sin embargo, correlaciones alrededor de .0 en la mayoría de los ítems, aunque las correlaciones parciales incrementaron ligeramente, lo que significa un efecto supresor de estas variables sobre la relación de los puntajes de la DS-14 y la salud mental (Aneshensel, 2012). Los ítems 2 y 4 de AN y los ítems 1 y 3 de IS, tendieron a mostrar un patrón correlacional diferente al resto de los ítems de sus subescalas, tanto respecto a la magnitud (alrededor de .0) como el signo (en algunos, asociación negativa), sugiriendo coincidencias con análisis previos y la inconsistencia de su validez.

\section{Discusión}

El objetivo del presente trabajo consistió en explorar las propiedades psicométricas de una adaptación del cuestionario DS-14 en una muestra de trabajadores mexicanos, incluyendo su estructura factorial, su consistencia interna, su invarianza de medición y su validez convergente con medidas de salud mental. Los resultados mostraron coeficientes de consistencia interna satisfactorios en consonancia con los estudios originales (Denollet, 1998, 2005) y con los estudios más recientes (Montero et al., 2017), donde se observan coeficientes similares (superiores a .80) en la mayoría de los casos, sobre todo para la dimensión de afectividad negativa, pues la inhibición social es usualmente la escala con mayor debilidad en consistencia interna, similar a lo sucedido en el presente estudio y en particular con la presencia de los ítems 1 y 3. En este sentido, una ventaja del presente estudio fue la utilización del coeficiente omega $(\omega)$, que supera algunas limitaciones del alfa de Cronbach (Dunn et al., 2014) lo que contribuye a confirmar la consistencia interna de la escala de manera universal, aún con medidas más robustas.

Asimismo, el análisis de invarianza factorial mostró ausencia de funcionamiento diferencial de la escala por sexo, lo que implica la confianza de medición equivalente entre hombres y mujeres. Esto permite observar que, aunque teóricamente se espera que la formación de la personalidad tiene una influencia innegable en relación al género y ello representa un impacto en la eficacia de la validez de su medición, la escala DS-14 no padece de este sesgo con los ítems utilizados. Se trata así de un aporte práctico relevante de este estudio ante la ausencia de exploración previa.

El análisis de dimensionalidad de la escala fue quizás el hallazgo más interesante, ya que aunque los índices de ajuste del modelo bifactorial original (afectividad negativa-inhibición social) fueron bastante satisfactorios (Tabla 3), también lo fue un modelo unidimensional, el cual a su vez mostró correlaciones parciales ligeramente superiores con los criterios de salud mental. Esto coincide con hallazgos previos ante el uso unidimensional de la escala de personalidad tipo D (Ferguson et al., 2009, Horwood et al., 2015; De Fruyt \& Denollet, 2002) y sobretodo con las fuertes correlaciones $(>.50)$ entre los componentes de afectividad negativa e inhibición social frecuentemente observadas en diversos estudios (Horwood et al., 2015; Montero et al., 2017; Spindler, Kruse, Zwisler, \& Pedersen, 2009; entre otros).

Son necesarias más investigaciones sobre la exploración unidimensional del constructo tipo D, pues sus implicaciones teórico-metodológicas y prácticas son relevantes. Por ejemplo, en el caso teórico implicaría un replanteamiento de algo que quizás es un constructo único que con riesgo de validez se ha partido en dos conceptos, cuya sinergia no ha sido definida claramente. En términos prácticos, debe considerarse que cuando se usa el criterio de clasificación original de «sujetos tipo D», se exige un puntaje alto para ambas escalas de manera bimodalcategórica (mayor a 10 puntos en cada escala en los estudios de Denollet, 2005), perdiendo así información y precisión en los casos en que la puntuación es ligeramente menor al punto de corte elegido en ambos constructos o en alguno de ellos (como sucede en el balance de medidas de tendencia central en el presente estudio, donde la media de afectividad negativa es de 
$M=9.14$ y de inhibición social es de $M=10.72$ (véase Tabla 4), teniendo así que categorizar radicalmente como «no tipo D» a las personas en estos casos, incluso en aquellas donde los puntajes son demasiado altos en solo una de las dos dimensiones, perdiendo fuerza en sus parámetros y desconocimiento de la contribución parcial del rasgo D en la salud al entrar en la misma clasificación rígida. De haber viabilidad teórica, debe vislumbrarse la posibilidad de un puntaje continuo (donde se puede tener poco o mucho de la «propiedad D») sumando el total de ítems de las dos escalas en la integración de un puntaje unidimensional que permite aprovechar la variabilidad del constructo. Esta perspectiva unidimensional haría también más parsimonioso su uso en la investigación multivariada y epidemiológica de la escala.

En el presente estudio, tanto el análisis no paramétrico como el paramétrico permitieron ubicar en particular 9 ítems que pueden conformar a la personalidad tipo $\mathrm{D}$ en un componente unidimensional como variable continua, con potencial para su estudio en investigaciones futuras. Los ítems menos robustos (que no significan totalmente inválidos), comprendieron dos relacionados con el desenvolvimiento social (1 y 3) que necesitan ser recodificados, uno de reticencia (10) y dos de afectividad negativa (2 y 4) (Tabla 1), los que al ser identificados en este primer estudio de validación en la cultura mexicana, se desconoce el origen de esta tendencia, aunque en el estudio de validación de la versión española (Montero et al., 2017) también los ítems 1, 2, 3 y 4 presentaron las cargas factoriales menos elevadas. Sin embargo, es necesario reiterar la necesidad de un planteamiento teórico viable para la integración sinérgica de la inhibición social y la afectividad negativa en un constructo único y estudios adicionales que confirmen o no su composición factorial, por lo que por ahora, se trata de un hallazgo que no debiera parecer tan extraño sabiendo las correlaciones de ambos constructos en estudios previos, pero que vislumbra la necesidad de más estudios futuros. Es importante recalcar que la versión original de la escala DS-14 mostró indicadores psicométricos satisfactorios y que la elección de 9 ítems se trata más bien de ítems con un desempeño apenas menor al óptimo, vislumbrando caminos futuros a una propuesta orientada a la parsimonia psicométrica sin descalificar el buen desempeño obtenido aquí por la escala original.

Con respecto a la validez con base a otras variables, la mayoría de las correlaciones con los componentes del síndrome de burnout y de la salud mental fueron significativas, con una magnitud de baja a moderada, algo inesperado pues se esperaban correlaciones de mayor magnitud. No obstante lo anterior, podrían ser suficientes para considerarse como evidencia adicional de la validez de la escala DS-14, sobre todo cuando el presente estudio se llevó a cabo con participantes aparentemente sanos y no pacientes enfermos, y se controló por los efectos de otros estresores psicosociales de alto impacto en los trastornos mentales según la literatura. $\mathrm{Al}$ respecto, vale la pena notar que el efecto de supresión hallado al controlar los estresores laborales significa la existencia de un mecanismo entre estas variables y el tipo de personalidad tipo D que implica por un lado, independencia en su contribución a la varianza de los indicadores de salud mental, pero también algún tipo de interacción entre variables de personalidad y del contexto laboral que necesita mayor estudio (Aneshensel, 2012).

En general, los resultados mostraron resultados favorables en el desempeño psicométrico de la Escala de Personalidad Tipo D (DS-14), por lo que se puede concluir que sus puntajes poseen validez y confiabilidad en una muestra mexicana. Sin embargo, es necesaria mayor investigación futura, donde se recomienda ampliar la muestra a otros sectores con propósitos de una representatividad más heterogénea e insistir en el contraste de la idoneidad de una estructura unidimensional versus la bidimensional, así como seguir trabajando en una versión más reducida sin perder sus alcances psicométricos.

Entre las limitaciones de este estudio se encuentran un tamaño de muestra limitado para ofrecer baremos de alcance poblacional, la naturaleza transversal del estudio, un proceso de adaptación con posibilidades de mayor rigor y la ausencia de indicadores de salud 
más objetivos (e.g., cardiovasculares), sin embargo, esta primera exploración de la validez de la escala DS14 en población mexicana ofrece un paso inicial en la investigación fértil de la relación entre la personalidad tipo D y la salud.

\section{Conflicto de intereses}

Los autores declaran no tener ningún conflicto de intereses en esta investigación.

\section{Responsabilidad ética}

El estudio no se sometió a revisión de comité de ética, pero cuenta con el seguimiento a los principios básicos de la declaración de Helsinki y se utilizó un consentimiento informado. La confidencialidad y privacidad de los participantes se resguardó de acuerdo a los lineamientos correspondientes

\section{Agradecimientos}

A todos los trabajadores participantes, a los alumnos de Licenciatura en Psicología de la UAEM de la materia de Psicosociología en el Trabajo, a las estudiantes de la Maestría en Psicología Ivonne Martínez y Miriam Penagos, y a los miembros tesistas del proyecto de factores psicosociales del trabajo por su apoyo en la recolección de datos.

\section{Referencias}

Akram, U., McCarty, K., Akram, A., Gardani, M., Tan, A., Villarreal, D., ... Mills, R. (2018). The relationship between type D personality and insomnia. Sleep Health: Journal of the National Sleep Foundation, 4(4), 360-363. doi: 10.1016/j.sleh.2018.04.005

Aneshensel, C. S. (2012). Theory-based data analysis for the social sciences. USA: Sage Publications.

Armon, G. (2014). Type D personality and job burnout: The moderating role of physical activity. Personality and Individual Differences, 58, 112-115. doi: 10.1016/j.pai d.2013.10.020

Asendorpf, J. B. (1993). Social inhibition: A generaldevelopmental perspective. In H. C. Traue \& J. W.
Pennebaker (Eds.), Emotion inhibition and health (pp. 80-99). Ashland, OH, US: Hogrefe \& Huber Publishers.

Asparouhov, T., \& Muthén, B. (2010). Weighted Least Squares Estimation with Missing Data. Recuperado de https://www.statmodel.com/download/Gstruc MissingRevision.pdf

Barnett, M., Ledoux, T., Garcini, L., \& Bakker, F. (2009). Type D personality and chronic pain: Construct and concurrent validity of the DS-14. Journal of Clinical Psychology in Medical Settings, 16(2), 194-199. doi: 10.1007/s10880-009-9152-0

Beaton, D. E., Bombardier, C., Guillemin, F., \& Ferraz, M. B. (2000). Guidelines for the process of cross-cultural adaptation of self-report measures. Spine, 25(24), 31863191. doi: 10.1097/00007632-200012150-00014

Bosco, F. A., Aguinis, H., Singh, K., Field, J. G., \& Pierce, C. A. (2015). Correlational effect size benchmarks. Journal of Applied Psychology, 100(2), 431-449. doi: $10.1037 / \mathrm{a} 0038047$

Bunevicius, A., Staniute, M., Brozaitiene, J., Stropute, D., Bunevicius, R., \& Denollet, J. (2013). Type D (distressed) personality and its assessment with the DS14 in Lithuanian patients with coronary artery disease. Journal of Health Psychology, 18(9), 12421251. doi: 10.1177/1359105312459098

Byrne, B. M. (2008). Testing for multigroup equivalence of a measuring instrument: A walk through the process. Psicothema, 20(4), 872-882. Recuperado de https://www.ncbi.nlm.nih.gov/pubmed/18940097

Byrne, B. M., Shavelson, R. J., \& Muthén, B. (1989). Testing for the equivalence of factor covariance and mean structures: The issue of partial measurement equivalence. Psychological Bulletin, 105, 456-466. doi: 10.1037//0033-2909.105.3.456

Cloninger, C. R., Przybeck, T. R., Svrakic, D. M., \& Wetzel, R. D. (1994). The Temperament and Character Inventory (TCI): A Guide to its Development and Use. Center for Psychobiology of Personality, St. Louis, MO.

Conraads, V. M., Denollet, J., De Clerck, L. S., Stevens, W. J., Bridts, C., \& Vrints, C. J. (2006). Type D personality is associated with increased levels of tumour necrosis factor (TNF)- $\alpha$ and TNF- $\alpha$ receptors in chronic heart failure. International Journal of Cardiology, 113(1), 34-38. doi: 10.1016/j.ijcard.2005.10.013 
Coyne, J. C., Jaarsma, T., Luttik, M. L., van Sonderen, E., van Veldhuisen, D. J., \& Sanderman, R. (2011). Lack of prognostic value of type $\mathrm{D}$ personality for mortality in a large sample of heart failure patients. Psychosomatic Medicine, 73(7), 557-562. doi: 10.1097/PSY.0b013e 318227 ac75

Costa, P. T. Jr., \& McCrae, R. R. (1990). Personality disorders and the five-factor model of personality. Journal of Personality Disorders, 4(4), 362-371. doi: 10.1521/pedi.1990.4.4.362

Costello, A. B., \& Osborne, J. W. (2005). Best practices in exploratory factor analysis: Four recommendations for getting the most from your analysis. Practical Assessment, Research \& Evaluation, 10(7), 1-9. doi: 10.4135/9781412995627.d8

De Fruyt, F., \& Denollet, J. (2002). Type D personality: A five-factor model perspective. Psychology and Health, 17(5), 671-683. doi: 10.1080/0887044 0290025858

Declaración de Helsinki (2013). Principios éticos para las investigaciones médicas en seres humanos. Recuperado de https://www.wma.net/es/policies-post/ declaracion-de-helsinki-de-la-amm-principios-eticospara-las-investigaciones-medicas-en-seres-humanos/

Denollet, J. (1991). Negative Affectivity and Repressive Coping: Pervasive Influence on Self-Reported Mood, Health, and Coronary-Prone Behavior. Psychosomatic Medicine, 53, 538-556. doi: 10.1097/00006842-199 109000-0005

Denollet, J., Rombouts, H., Gillebert, T. C., Brutsaert, D. L., Sys, S.U., \& Stroobant, N. (1996). Personality as independent predictor of long-term mortality in patients with coronary heart disease. The Lancet, 347(8999), 417-421. doi: 10.1016/S0140-6736(96)90007-0

Denollet, J. (1998). Personality and coronary heart disease: the type-D scale-16. Annals of Behavioral Medicine, 20, 209-215. doi: 10.1007/BF02884962

Denollet, J., Vaes, J., \& Brutsaert, D. L. (2000). Inadequate response to treatment in coronary heart disease: adverse effects of type D personality and younger age on 5-year prognosis and quality of life. Circulation, 102(6), 630-635. doi: 10.1161/01. CIR.102.6.630
Denollet, J. (2000). Type D personality. A potential risk factor refined. Journal of Psychosomatic Research, 49(2000), 255-266. Recuperado de https://www.ncbi. nlm.nih.gov/pubmed/11119782

Denollet, J., Conraads, V. M., Brutsaert, D. L., De Clerck, L. S., Stevens, W. J., \& Vrints, C. J. (2003). Cytokines and immune activation in systolic heart failure: the role of Type D personality. Brain, Behavior, and Immunity, 17(4), 304-309. doi: 10.1016/S0889-15 (03)00060-6

Denollet, J. (2005). DS-14: Standard assessment of negative affectivity, social inhibition, and type D personality. Psychosomatic Medicine, 67, 89-97. doi: 10.1097/01.psy.0000149256.81953.49

Denollet, J., Pedersen, S. S., Vrints, C. J., \& Conraads, V. M. (2006). Usefulness of type D personality in predicting five-year cardiac events above and beyond concurrent symptoms of stress in patients with coronary heart disease. The American Journal of Cardiology, 97(7), 970-973. doi: 10.1016/j.amjcard. 2005.10.035

Denollet, J. \& Conraads, V.M. (2011). Type D personality and vulnerability to adverse outcomes in heart disease. Cleveland Clinical Journal of Medicine, 78(1). doi: 10.3949/ccjm.78.s1.02

Dunn, T. J., Baguley, T., \& Brunsden, V. (2014). From alpha to omega: A practical solution to the pervasive problem of internal consistency estimation. British Journal of Psychology, 105(3), 399-412. doi: 10.1111/bjop.12046

Emons, W. H., Meijer, R. R., \& Denollet, J. (2007). Negative affectivity and social inhibition in cardiovascular disease: evaluating type-D personality and its assessment using item response theory. Journal of Psychosomatic Research, 63(1), 27-39. doi: 10.1016/j.jpsychores.2 007.03.010

Eysenck, H. J. (1967). The biological basis of personality. Springfield, IL: Thomas.

Eysenck, H. J., \& Eysenck, M. W. (1987). Personalidad y diferencias individuales. Madrid: Pirámide.

Ferguson, E., Williams, L., O’connor, R. C., Howard, S., Hughes, B. M., Johnston, D.W., ... O’carroll, R. E. (2009). A taxometric analysis of type-D personality. Psychosomatic Medicine, 71(9), 981986. doi: 10.1097/PSY.0b013e3181bd888b 
Friedman, M., \& Rosenman, R. H. (1959). Association of specific overt behavior pattern with blood and cardiovascular findings: blood cholesterol level, blood clotting time, incidence of arcus senilis, and clinical coronary artery disease. Journal of the American Medical Association, 169(12), 1286-1296. doi: 10.1001/jama.1959.03000290012005

González, N. L., Juárez, A., Camacho, A., Noriega, L. H., \& Escobedo, J. P. (2015). Validez factorial y confiabilidad del cuestionario del contenido del trabajo (jcq: job content questionnarie) en empleados del sector salud, México. En A. Juárez-García. Investigaciones psicométricas de escalas psicosociales en trabajadores mexicanos (pp. 3564). Morelos: Plaza y Valdez Editores.

Gramer, M., Haar, J., \& Mitteregger, M. (2018). Type D personality and cardiovascular reactivity in active performance situations: Gender and task-specific influences. Personality and Individual Differences, 132, 74-78. doi: 10.1016/j.paid.2018. 05.027

Grande, G., Jordan, J., Kümmel, M., Struwe, C., Schubmann, R., Schulze, F., ... Herrmann-Lingen, C. (2004). Evaluation of the German Type D Scale (DS14) and prevalence of the Type D personality pattern in cardiological and psychosomatic patients and healthy subjects. Psychotherapie, Psychosomatik, Medizinische Psychologie, 54(11), 413-422. doi: 10.1 055/s-2004-828376

Gray, J. A. (1982). The Neuropsychology of Anxiety: An Enquiry into the Function of the Septohippocampal System. Oxford University Press, New York.

Habra, M. E., Linden, W., Anderson, J. C., \& Weinberg, J. (2003). Type D personality is related to cardiovascular and neuroendocrine reactivity to acute stress. Journal of Psychosomatic Research, 55(3), 235-245. doi: 10.1016/S0022-3999(02)00553-6

Hemker, B. T., Sijtsma, K., \& Molenaar, I. W. (1995). Selection of unidimensional scales from a multidimensional item bank in the polytomous Mokken IRT model. Applied Psychological, Measurement, 19, 337-352. doi: 10.1177/014662169501900404

Horwood, S., Anglim, J., \& Tooley, G. (2015). Type D personality and the Five-Factor Model: A facet-level analysis. Personality and Individual Differences, 83, 50-54. doi: 10.1016/j.paid.2015.03.041
Jandackova, V. K., Koening, J., Jarczok, M. N., Fischer, J. E., \& Thayer, J. F. (2017). Potential biological pathways linking type-D personality and poor health: A crosssectional investigation. PLOS ONE, 12(4), 1-13. doi: 10.1371/journal.pone.0176014

Juárez-García, A., Merino-Soto, C., Fernández, M., Flores, C., Caraballo, M., \& Camacho, C. (2018). Validación Transcultural y Funcionamiento Diferencial del MBIGS en tres Países Latinoamericanos. Artículo enviado para dictamen.

Kline, R. B. (2010). Principles and practice of structural equation modeling (3rd ed.). New York: The Guilford Press.

Kupper, N., Pedersen, S. S., Höfer, S., Saner, H., Oldridge, N., \& Denollet, J. (2013). Cross-cultural analysis of Type D (distressed) personality in 6222 patients with ischemic heart disease: A study from the International HeartQoL Project. International Journal of Cardiology, 166(2), 327-333. doi: 10.1016/j.ijcar d.2011.10.084

Lahey, B. B. (2009). Public health significance of neuroticism. American Psychologist, 64(4), 241256. doi:10.1037/a0015309. doi: 10.1037/a0015309

Li, C. H. (2016). Confirmatory factor analysis with ordinal data: Comparing robust maximum likelihood and diagonally weighted least squares. Behavior Research Methods, 48(3), 936-949. doi: 10.3758/s1 3428-015-0619-7

Lin, I. M., Wang, S. Y., Chu, I. H., Lu, Y. H., Lee, C. S., Lin, T. H., \& Fan, S. Y. (2017). The association of type D personality with heart rate variability and lipid profiles among patients with coronary artery disease. International Journal of Behavioral Medicine, 24, 101-109. doi: 10.1007/s12529-016-9571-x

Madsen, I. E., Nyberg, S. T., Hanson, L. M., Ferrie, J. E., Ahola, K., Alfredsson, L., ... Chastang, J. F. (2017). Job strain as a risk factor for clinical depression: systematic review and meta-analysis with additional individual participant data. Psychological Medicine, 47(8), 13421356. doi: 10.1017/S003329171600355X

Maslach, C., Jackson, S. E., \& Leiter, M. P. (2010). Maslach Burnout Inventory: Manual and Non-reproducible Instrument and Scoring Guides. Mind Garden. 
Medina-Mora, M. E., Padilla, G. P., Campillo-Serrano, C., Mas, C. C., Ezban, M., Caraveo, J., \& Corona, J. (1983). The factor structure of the GHQ: a scaled version for a hopital's general practice service in Mexico. Psychological Medicine, 13(2), 355-361. doi: $10.1017 /$ S0033291700050984

Messing, K., Punnett, L., Bond, M., Alexanderson, K., Pyle, J., Zahm, S., ... de Grosbois, S. (2003). Be the fairest of them all: challenges and recommendations for the treatment of gender in occupational health research. American Journal of Industrial Medicine, 43(6), 618-629. doi: 10.1002/ajim.10225

Mokken, R. J. (1997). Nonparametric models for dichotomous responses. In W. J. van der Linden \& R. K. Hambleton (Eds.), Handbook of Modern Item Response Theory. New York: Springer

Molenaar, I. (1997). Nonparametric models for polytomous responses. In W. J. van der Linden \& R. K. Hambleton (Eds.), Handbook of Modern Item Response Theory. New York: Springer.

Mols, F., \& Denollet, J. (2010). Type D personality in the general population: a systematic review of health status, mechanisms of disease, and work-related problems. Health and Quality of Life Outcomes, 8(1), 9. doi: 10.1186/1477-7525-8-9

Mols, F., Thong, M. S., van de Poll-Franse, L. V., Roukema, J. A., \& Denollet, J. (2012). Type D (distressed) personality is associated with poor quality of life and mental health among 3080 cancer survivors. Journal of Affective Disorders, 136(1-2), 26-34. doi: 10.1016/j.jad.2011.08.034

Montero, I., \& León, O. G. (2007). A guide for naming research studies in Psychology. International Journal of Clinical and Health Psychology, 7(3). Recuperado de http://www.aepc.es/ijchp/GNEIP 07_es.pdf

Montero, P., Bermúdez, J., \& Rueda, B. (2017). Adaptación al castellano de la Escala DS-14 («Type D Scale-14») para la medida de la personalidad tipo D. Revista de Psicopatología y Psicología Clínica, 22(1). doi: 10.5944/rppc.vol.22.num.1.2 017.16585

Montero, P., Rueda, B. B., \& Bermúdez, J. (2012). Relación de la personalidad tipo D y el agotamiento vital con las emociones negativas y el ajuste psicológico a la enfermedad cardiaca. Revista de Psicopatología y Psicología Clínica, 17(2), 93-106. doi: 105944/rppc.vol.17.num.2.2012.11318

Muthén, B. (1984). A general structural equation model with dichotomous, ordered categorical, and continuous latent variable indicators. Psychometrika, 49(1), 115-132.

Pedersen, S. S., \& Denollet, J. (2003). Type D personality, cardiac events, and impaired quality of life: a review. European Journal of Cardiovascular Prevention \& Rehabilitation, 10(4), 241-248. doi: 10.1097/01.hjr.0000085246.65733.06

Pedersen, S. S., \& Denollet, J. (2004). Validity of the Type $\mathrm{D}$ personality construct in Danish post-MI patients and healthy controls. Journal of Psychosomatic Research, 57(3), 265-272. doi: 10.1016/S0022-399 9(03)00614-7

Pedersen, S. S., Theuns, D. A., Muskens-Heemskerk, A., Erdman, R. A., \& Jordaens, L. (2007). Type-D personality but not implantable cardioverterdefibrillator indication is associated with impaired health-related quality of life 3 months postimplantation. Europace, 9(8), 675-680. doi: 10.1093/ europace/eum041

Pedersen, S. S., van Domburg, R. T., Theuns, D. A., Jordaens, L., \& Erdman, R. A. (2004). Type D personality is associated with increased anxiety and depressive symptoms in patients with an implantable cardioverter defibrillator and their partners. Psychosomatic Medicine, 66(5), 714-719. doi: 10.1097/01.psy.00001 32874.52202.21

Polman, R., Borkoles, E., \& Nicholls, A. R. (2010). Type $\mathrm{D}$ personality, stress, and symptoms of burnout: The influence of avoidance coping and social support. British Journal of Health Psychology, 15(3), 681696. doi: 10.1348/135910709x479069

Putnick, D. L., \& Bornstein, M. H. (2016). Measurement Invariance Conventions and Reporting: The State of the Art and Future Directions for Psychological Research. Developmental review, 41, 71-90. doi: 10.1016/j.dr. 2016.06.004

Rosenfield, S., \& Mouzon, D. (2013). Gender and mental health. In Handbook of the sociology of mental health (pp. 277-296). Springer, Dordrecht. 
Ruch, W. (1992). Pavlov's types of nervous system, Eysenck's typology and the Hippocrates-Galen temperaments: An empirical examination of the asserted correspondence of three temperament typologies. Personality and Individual Differences, 13(12), 1259-1271. doi: 10.1016/0191-8869(92) 90168-O

Sass, D. A., Schmitt, T. A., \& Marsh, H. W. (2014). Evaluating model fit with ordered categorical data within a measurement invariance framework: A comparison of estimators. Structural Equation Modeling: A Multidisciplinary Journal, 21(2), 167180. doi: 10.1080/10705511.2014.882658

Sher, L. (2005). Type D personality: the heart, stress, and cortisol. Qjm, 98(5), 323-329. doi: 10.1093/qjmed/ hci064

Spindler, H., Kruse, C., Zwisler, A., \& Pedersen, S. (2009). Increased anxiety and depression in Danish cardiac patients with a type D personality: Crossvalidation of the type D scale (DS14). International Journal of Behavioral Medicine, 16(2), 98-107. doi: 10.1007/s12529-009-9037-5

Stansfeld, S., \& Candy, B. (2006). Psychosocial work environment and mental health a meta-analytic review. Scandinavian Journal of Work, Environment \& Health, 23(6), 443-462. doi: 10.5271/sjweh.1050

Stelmack, R. M., \& Stalikas, A. (1991). Galen and the humour theory of temperament. Personality and Individual Differences, 12(3), 255-263. doi: 10.101 6/0191-8869(91)90111-N

Stochl, J., Jones, P. B., \& Croudace, T. J. (2012). Mokken scale analysis of mental health and well-being questionnaire item responses: a non-parametric IRT method in empirical research for applied health researchers. BMC medical research methodology, 12, 74. https://doi.org/10.1186/1471-2288-12-74

Svansdottir, E., Karlsson, H. D., Gudnason, T., Olason, D. T., Thorgilsson, H., Sigtryggsdottir, U., ... Denollet, J. (2012). Validity of Type D personality in Iceland: association with disease severity and risk markers in cardiac patients. Journal of Behavioral Medicine, 35(2), 155-166. doi: 10.1007/s10865-011-9337-5

Theorell, T., Hammarström, A., Aronsson, G., Bendz, L. T., Grape, T., Hogstedt, C., ... Hall, C. (2015). A systematic review including meta-analysis of work environment and depressive symptoms. BMC public health, 15(1), 738. doi: 10.1186/s12889-015-1954-4

Van der Ark, L. A. (2007). Mokken Scale Analysis in R. Journal of Statistical Software, 20(11), 1-19. doi: 10.18637/jss.v020.i11

Watson, D., Clark, L. A., \& Tellegen, A. (1988). Development and validation of brief measures of positive and negative affect: The PANAS scales. Journal of Personality and Social Psychology, 54, 1063-1070. doi: 10.1037/0022-35 14.54.6.1063

Weng, C. Y., Denollet, J., Lin, C. L., Lin, T. K., Wang, W. C., Lin, J. J., Wong, S. S., \& Mols, F. (2013). The validity of the type $\mathrm{D}$ construct and its assessment in Taiwan. BMC Psychiatry, 13(46), 1-9. doi: 10.1186/ 1471-244X-13-46

Whitehead, D. L., Perkins-Porras, L., Strike, P. C., Magid, K., \& Steptoe, A. (2007). Cortisol awakening response is elevated in acute coronary syndrome patients with type-D personality. Journal of Psychosomatic Research, 62(4), 419-425. doi: 10.10 16/j.jpsychores.2006.11.005

Williams, L., O’Carroll, R. E., \& O’Connor, R. C. (2009). Type D personality and cardiac output in response to stress. Psychology and Health, 24(5), 489-500. doi: 10.1080/08870440701885616

Williams, L., O’Connor, R. C., Howard, S., Hughes, B. M., Johnston, D. W., Hay, J. L., ... Grealy, M. A. (2008). Type-D personality mechanisms of effect: the role of health-related behavior and social support. Journal of Psychosomatic Research, 64(1), 63-69. doi: 10.101 6/j.jpsychores.2007.06.008 


\section{Arturo Juárez-García}

Centro de Investigación Transdisicplinar en Psicología, Universidad Autónoma de Estado de Morelos, México.

Doctor en Psicología, cuenta con publicaciones sobre la relación entre factores psicosociales y la salud mental y física, así como las propiedades psicométricas de escalas utilizadas en trabajadores. Su línea de investigación trata sobre la Psicología de la Salud Ocupacional.

ORCID: 0000-0003-3264-679X

Autor corresponsal: arturojuarezg@hotmail.com

\section{César Merino-Soto}

Universidad de San Martín de Porres

Psicólogo, investigador en el Instituto de Investigación de Psicología (Universidad de San Martín de Porres); orientado en psicometría y metodología cuantitativa.

ORCID: 0000-0002-1407-8306

sikayax@yahoo.com.ar

Javier Neri Uribe

Facultad de Contaduría, Administración e Informática, Universidad Autónoma del Estado de Morelos.

Doctor en Psicología, cuenta con investigaciones en clima organizacional, inteligencia emocional, síndrome de burnout y salud mental. Su línea de investigación se centra en el estudio del trabajo y la psicología en las organizaciones.

javier_neri02@hotmail.com 